\title{
Thread-Level Speculation on a CMP Can Be Energy Efficient *
}

\author{
Jose Renau ${ }^{\dagger}$ Karin Strauss Luis Ceze Wei Liu Smruti Sarangi James Tuck Josep Torrellas \\ ${ }^{\dagger}$ Dept. of Computer Engineering, University of California Santa Cruz \\ http://masc.soe.ucsc.edu \\ Dept. of Computer Science, University of Illinois at Urbana-Champaign \\ http://iacoma.cs.uiuc.edu
}

\begin{abstract}
Chip Multiprocessors (CMP) with Thread-Level Speculation (TLS) have become the subject of intense research. However, TLS is suspected of being too energy inefficient to compete against conventional processors. In this paper, we refute this claim. To do so, we first identify the main sources of dynamic energy consumption in TLS. Then, we present simple energy-saving optimizations that cut the energy cost of TLS by over $60 \%$ on average with minimal performance impact. The resulting TLS CMP, populated with four 3-issue cores, speeds-up full SPECint 2000 codes by 1.27 on average, while keeping the fraction of the chip's energy consumption due to TLS to only $20 \%$. Compared to a 6-issue superscalar at the same frequency, the TLS CMP is on average faster, while consuming only $85 \%$ of its total on-chip power.
\end{abstract}

\section{Introduction}

Substantial research effort is currently being devoted to speeding up hard-to-parallelize non-numerical applications such as SPECint codes. Designers build sophisticated out-of-order processors, with carefully-tuned execution engines and memory subsystems. Unfortunately, these systems tend to combine high design complexity with diminishing performance returns, motivating the search for design alternatives.

One such alternative is Thread-Level Speculation (TLS) on a Chip Multiprocessor (CMP) [9, 10, 13, 21, 22, 25, 26]. Under TLS, these hard-to-analyze applications are partitioned into tasks, which are then optimistically executed in parallel, hoping that no data or control dependence will be violated. Special hardware support monitors the tasks' control flow and data accesses, and detects violations at run time. Should one occur, the hardware transparently rolls back the incorrect tasks and, after repairing the state, restarts them.

Published results show that TLS CMPs can speed up difficult nonnumerical applications. This is significant because CMPs are attractive platforms; they provide a low-complexity, energy-efficient architecture, and have a natural advantage for explicitly-parallel codes.

However, TLS is suspected of being too energy inefficient to seriously challenge conventional processors. The rationale is that aggressive speculative execution is not the best course at a time when processors are primarily constrained by energy and power issues. Our initial experiments, shown in Figure 1, appear to agree: assuming constant frequency, a high-performance TLS CMP with four 3-issue

*This work was supported in part by the National Science Foundation under grants EIA-0072102, EIA-0103610, CHE-0121357, and CCR-0325603; DARPA under grant NBCH30390004; DOE under grant B347886; and gifts from IBM and Intel.

Permission to make digital or hard copies of all or part of this work for personal or classroom use is granted without fee provided that copies are not made or distributed for profit or commercial advantage and that copies bear this notice and the full citation on the first page. To copy otherwise, or republish, to post on servers or to redistribute to lists, requires prior specific permission and/or a fee. ICS'05, June 20-22, Boston, MA, USA. Copyright (C) 2005 , ACM 1-59593-167-8/06/2005...\$5.00 cores is on average faster than a 6-issue superscalar for SPECint codes, but consumes on average $15 \%$ more on-chip power.

In this paper, we refute the claim that TLS is energy inefficient. This paper is the first one to show that, perhaps contrary to commonly-held views, a TLS CMP can be a very desirable design for high-performance, power-constrained processors, even under the very challenging SPECint codes.

Fundamentally, the energy cost of TLS can be kept modest by using a lean TLS CMP microarchitecture and by minimizing wasted TLS work. Then, such a TLS CMP provides a better energyperformance trade-off than a wider-issue superscalar simply because, as the size of the processor structures increases, energy scales superlinearly and performance sublinearly.

This paper offers three contributions. The first one is to identify and quantify the main sources of energy consumption in TLS. These sources are task squashing, hardware structures in the cache hierarchy for data versioning and dependence checking, additional traffic in the memory subsystem due to the same two effects, and additional instructions induced by TLS.

The second contribution is to present and evaluate simple energysaving optimizations for TLS. They are based on reducing the number of checks, reducing the cost of individual checks, and eliminating work with low performance returns. These optimizations cut the energy cost of TLS by over $60 \%$ on average, with minimal performance impact.

The third contribution is to show that the resulting TLS CMP can provide a very desirable energy-performance trade-off, even for SPECint codes. Specifically, a TLS CMP with four 3-issue cores speeds-up full SPECint 2000 codes by 1.27 on average, while keeping the fraction of the chip's energy consumption due to TLS to only $20 \%$. Moreover, compared to a 6-issue superscalar at the same frequency, the TLS CMP is on average faster, while consuming only $85 \%$ of its total on-chip power. Finally, we expect better results for floating point, multimedia, or more parallel codes.

This paper is organized as follows: Section 2 provides a background; Section 3 examines why TLS consumes more energy; Section 4 outlines our TLS architecture and compiler; Section 5 describes simple optimizations to save energy in TLS; Sections 6 and 7 present our methodology and evaluation; and Section 8 lists related work.

\section{Thread-Level Speculation (TLS)}

Overview. In TLS, a sequential program is divided into tasks, which are then executed in parallel, hoping not to violate sequential semantics. The sequential code imposes a task order and, therefore, we use the terms predecessor and successor tasks. The safe (or nonspeculative) task precedes all speculative tasks. As tasks execute, special hardware support checks that no cross-task dependence is violated. If any is, the incorrect tasks are squashed, any polluted state is repaired, and the tasks are re-executed.

Cross-Task Dependence Violations. Data dependences are typically monitored by tracking, for each individual task, the data written and the data read with exposed reads. An exposed read is a read that is not preceded by a write to the same location within the same task. A 


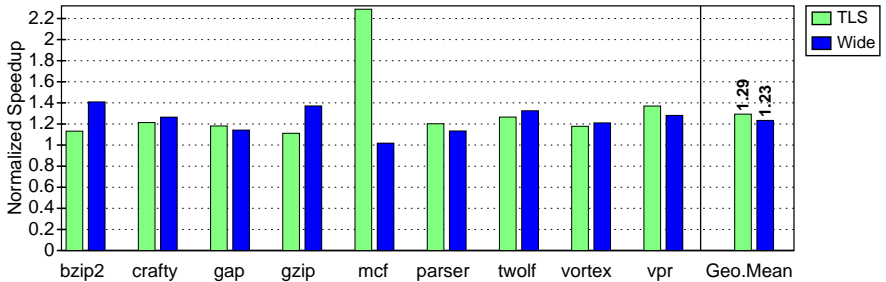

(a)

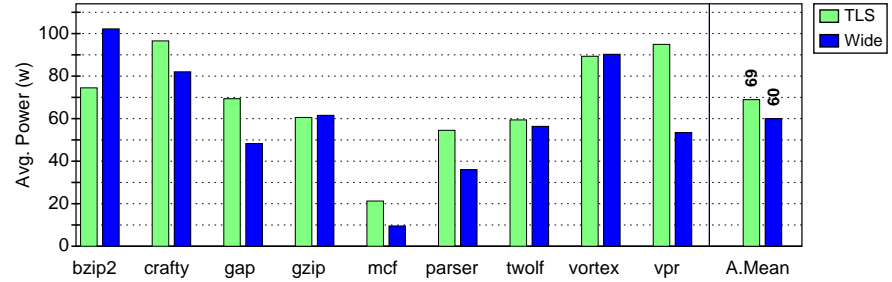

(b)

Figure 1: Comparing the performance (a) and power (b) of a high-performance TLS CMP with four 3-issue cores (TLS) and a 6-issue superscalar (Wide) for SPECint codes. The experiments, which are described in detail later, use a constant frequency. The bars in (a) are normalized to the performance of a 3-issue superscalar.

data dependence violation occurs when a task writes a location that has been read by a successor task with an exposed read. A control dependence violation occurs when a task is spawned in a mispredicted branch path. Dependence violations lead to task squashes, which involve discarding the work produced by the task.

State Buffering. Stores issued by a speculative task generate speculative state that cannot be merged with the safe state of the program because it may be incorrect. Such state is stored separately, typically in the cache of the processor running the task. If a violation is detected, the state is discarded. Otherwise, when the task becomes non-speculative, the state is allowed to propagate to memory. When a non-speculative task finishes execution, it commits. Committing informs the rest of the system that the state generated by the task is now part of the safe program state.

Data Versioning. A task has at most a single version of any given variable. However, different speculative tasks that run concurrently in the machine may write to the same variable and, as a result, produce different versions of the variable. Such versions must be buffered separately. Moreover, readers must be provided the correct versions. Finally, as tasks commit in order, data versions need to be merged with the safe memory state also in order.

Multi-Versioned Caches. A cache that can hold state from multiple tasks is called multi-versioned [5, 8, 22]. There are two performance reasons why multi-versioned caches are desirable: they avoid processor stall when tasks are imbalanced, and enable lazy commit.

If tasks have load imbalance, a processor may finish a task and the task still be speculative. If the cache can only hold state for a single task, the processor has to stall until the task becomes safe [6]. An alternative is to move the task's state to some other buffer, but this complicates the design. Instead, the cache can retain the state from the old task and allow the processor to execute another task. If so, the cache has to be multi-versioned.

Lazy commit [17] is an approach where, when a task commits, it does not eagerly merge its cache state with main memory through ownership requests [22] or write backs [10]. Instead, the task simply passes the commit token to its successor. Its state remains in the cache and is lazily merged with main memory later, usually as a result of cache line replacements. This approach improves performance because it speeds up the commit operation. However, it requires multiversioned caches.

Tagging Multi-Versioned Caches. Multi-versioned caches typically require that we tag each cache line with a version ID, which records what task the line belongs to. Intuitively, such version ID could be the long global ID of the task. However, to save space, it is best to translate global task IDs into some arbitrary Local IDs (LIDs) that are much shorter [22]. These LIDs are used only locally in the cache, to tag cache lines. Their translations into global IDs are kept in a small, per-cache table that we call LID Table. Each cache has a different LID Table.

Architecture and Environment Considered. While TLS can be supported in different ways, we use a CMP because it is a lowcomplexity, energy-efficient platform. Moreover, to maximize the use of commodity hardware, our CMP has no special hardware support for inter-processor register communication. Processors can only communicate via the memory system. In addition, to gain usability, our speculative tasks are generated automatically by a TLS compiler. Finally, we concentrate on SPECint 2000 applications because they are very challenging to speed-up.

\section{Sources of Energy Consumption in TLS}

Enhancing a $W$-wide superscalar into a CMP with several $W$-wide cores and TLS support causes the energy consumption to increase. We loosely call the increase the energy cost of $T L S\left(\Delta E_{T L S}\right)$. In practice, a portion of $\Delta E_{T L S}$ simply comes from having multiple cores and caches on chip, and from inefficiencies of parallel execution. However, most of $\Delta E_{T L S}$ is due to TLS-specific sources. We are interested in the latter.

We propose to classify TLS-specific sources of energy consumption into four main groups: (1) task squashing, (2) hardware structures in the cache hierarchy needed to support data versioning and dependence checking, (3) additional traffic in the memory system due to these same two effects, and (4) additional dynamic instructions induced by TLS. These sources are detailed in Table 1 .

\begin{tabular}{|c|c|c|}
\hline \multicolumn{2}{|c|}{ TLS-Specific Source } & Optimization \\
\hline \multirow[t]{2}{*}{ Task squashing } & $\begin{array}{l}\text { Work of the tasks that } \\
\text { get squashed }\end{array}$ & \multirow[t]{2}{*}{ StallSq, TaskOpt } \\
\hline & Task squash operations & \\
\hline \multirow{2}{*}{$\begin{array}{l}\text { Hardware structures } \\
\text { in the cache hierarchy } \\
\text { for data versioning and } \\
\text { dependence checking }\end{array}$} & $\begin{array}{l}\text { Storage \& logic for data } \\
\text { version IDs and access bits }\end{array}$ & Indirect \\
\hline & Tag-group operations & NoWalk \\
\hline \multirow{3}{*}{$\begin{array}{l}\text { Traffic due to data } \\
\text { versioning and } \\
\text { dependence checking }\end{array}$} & $\begin{array}{l}\text { Evictions and misses due } \\
\text { to higher cache pressure }\end{array}$ & - \\
\hline & $\begin{array}{l}\text { Selection \& combination } \\
\text { of multiple versions }\end{array}$ & \multirow{2}{*}{ TrafRed } \\
\hline & $\begin{array}{l}\text { Fine-grain data } \\
\text { dependence tracking }\end{array}$ & \\
\hline \multirow{2}{*}{$\begin{array}{l}\text { Additional dynamic } \\
\text { instructions induced } \\
\text { by TLS }\end{array}$} & $\begin{array}{l}\text { Side-effects of breaking } \\
\text { the code into tasks }\end{array}$ & \multirow[t]{2}{*}{ TaskOpt } \\
\hline & TLS-specific instructions & \\
\hline
\end{tabular}

Table 1: Main TLS-specific sources of energy consumption.

\subsection{Task Squashing}

An obvious TLS source of energy consumption is the work of tasks that ultimately get squashed. In the TLS CMP that we evaluate in Section 7, 22.6\% of all graduated instructions belong to such tasks. Note, however, that not all such work is wasted: a squashed task may bring useful data into the caches or train the branch predictor.

The actual squash operation also consumes energy: a squash signal is sent to the target processor, and a hardware finite-state machine (FSM) is activated to repair the state. In our system, such repair only involves restoring the program counter and stack pointer, and setting the task's LID Table entry to invalid. In our system, the frequency of squashes is only 1 per 3211 instructions on average. Consequently, the total energy consumed by the actual squash operations is negligible. 


\subsection{Hardware Structures for Data Versioning and Dependence Checking}

The two most characteristic operations of TLS systems are maintaining data versioning and performing dependence checking. These operations are largely supported in the cache hierarchy. Data versioning is needed when the cache hierarchy can hold multiple versions of the same datum. Such versions appear when speculative tasks have WAW or WAR dependences with predecessor tasks. The version created by the speculative task is buffered, typically in the processor's cache. If multiple speculative tasks co-exist in the same processor, a cache may have to hold multiple versions of the same datum. In such cases, data versions are identified by tagging the cache lines with a version ID - in our case an LID (Section 2).

To perform dependence checking, caches record how each datum was accessed. Typically, this is supported by augmenting each cached datum with two access bits: an exposed-read and a write bit. They are set on an exposed read and a write, respectively.

The LID and access bits are read or updated in hardware in a variety of cache access operations. For example, on an external access to a cache, the (translated) LID of an address-matching line in the cache is compared to the ID of the incoming message. From the comparison and the value of the access bits, the cache may conclude that a violation occurred, or can instead supply the data normally.

A distinct use of these TLS structures is in tag-group operations. They involve changing the tag state of groups of cache lines. There are three main cases. First, when a task is squashed, all its cache lines need to be eventually invalidated. Second, in eagercommit systems [6], when a task commits, all its dirty cache lines are merged with main memory through write backs [10] or ownership requests [22]. Finally, in lazy-commit systems, when a cache has no free LIDs left, it needs to recycle one. This is typically done by selecting a long-committed task and writing back all its dirty cache lines to memory. Then, that task's LID becomes free and can be reassigned.

These TLS tag-group operations often induce significant energy consumption. Specifically, for certain operations, some schemes use a hardware FSM that, periodically and in the background, repeatedly walks the tags of the cache. For example, to recycle LIDs in [17], a FSM periodically selects the LID of a committed task from the LID Table, walks the cache tags writing back to memory the dirty lines of that task, and finally frees up the LID. The FSM operates in the background eagerly, using free cache cycles. Other schemes perform similar hardware walks of tags while stalling the processor to avoid causing races. For example, to commit a task in [22], a special hardware module sequentially requests ownership for a group of cache lines whose addresses are stored in a buffer. Since the processor stalls, execution takes longer and, therefore, consumes more energy. Finally, some schemes use "one-shot" hardware signals that can change the tag state of a large group of lines in a handful of cycles. For example, this is done to invalidate the lines of a squashed task. Such hardware is reasonable when the cache can hold data for only a single or very few speculative tasks $[5,9,22]$. However, in caches with many versions, it is likely to adversely affect the cache access time. For example, in our system, we use 6-bit LIDs per cache line. A "one-shot" clear of the valid bit of all the lines belonging to a given task would require to keep, for each line tag, 6 NXOR gates that feed into one (possibly cascaded) AND gate. Having such logic per tag entry is likely to slow down the common case of a plain cache access, and result in longer, more energy-consuming executions.

\subsection{Additional Traffic for Data Versioning and Dependence Checking}

A TLS CMP system generates more traffic than a superscalar. The increase is $460 \%$ in our system (Section 7). While some of the increase is the result of parallel execution, there are three main TLS-specific sources of additional traffic (Table 1).

One reason is that caches do not work as well. Caches often have to retain lines from older tasks that ran on the processor and are still speculative. Only when such tasks become safe can the lines be evicted. As a result, there is less space in the cache for data that may be useful to the task currently running locally. This higher cache pressure increases evictions of useful lines and subsequent misses.

The presence of multiple versions of the same line in the system also causes additional messages. Specifically, when a processor requests a line, multiple versions of it may be provided, and the coherence protocol then selects what version to use. Similarly, when a committed version of a line is to be evicted to L2, the protocol first invalidates all the other cached versions of the line that are older they cannot remain cached anymore.

Finally, it is desirable that the speculative cache coherence protocol track dependences at a fine grain, which creates additional traffic. To see why, recall that these protocols typically track dependences by using the write and exposed-read bits. If this access information is kept per line, lines that exhibit false sharing may appear to be involved in data dependence violations and, as a result, cause squashes [5]. For this reason, many TLS proposals keep some access information at a finer grain, such as per word. Unfortunately, per-word dependence tracking may induce higher traffic: a distinct message (such as an invalidation) may need to be sent for every word of the line.

\subsection{Additional Dynamic Instructions Due to TLS}

TLS systems with compiler-generated tasks such as ours often execute more dynamic instructions than non-TLS systems. This is the case even counting only tasks that are not squashed. In our system, the increase is $12.5 \%$. These additional instructions come from two sources (Table 1): side-effects of breaking the code into tasks and, less importantly, TLS-specific instructions.

The first source dominates. It accounts for $88.3 \%$ of the increase. One reason for this source is that conventional compiler optimizations are not very effective at optimizing code across task boundaries. Therefore, TLS code quality is lower than non-TLS code. In addition, in CMPs where processors communicate only through memory, the compiler must spill registers across task boundaries.

TLS-specific instructions are the other source. They include task spawn and commit instructions. The spawn instruction sends some state from one processor to another. Task commit in lazy implementations sends the commit token between processors [17]. These instructions contribute with $11.7 \%$ of the instruction increase.

\section{TLS Architecture and Compiler}

Before we examine ways to reduce TLS energy consumption, it is helpful to outline the high-performance TLS CMP architecture and compiler that we use as baseline in our work. More details can be found in $[18,19,27]$.

\subsection{TLS CMP Architecture}

The CMP connects four modest-issue processors in a virtual ring. Each processor has a private, multi-versioned L1. The ring is also connected to a small, multi-versioned victim cache. Finally, there is a plain, shared L2 that only holds safe data (Figure 2-(a)). We use a ring interconnect to minimize races in the coherence protocol. The victim cache is included to avoid the more expensive alternative of designing a multi-versioned L2. Figures 2-(b) and (c) show the extensions required by TLS to the processors, L1s, and victim cache. Each structure shows its fields in the form bit_count:field_name.

Each processor has an array of TaskHolders, which are hardware structures that hold some state for the tasks that are currently loaded on-chip (Figure 2-(b)). Each TaskHolder contains the task's LID, its spawn address (PC), its stack pointer (SP), and a few additional bits that will be discussed later. The register state is kept on the stack.

A copy of the LID for the task currently going through rename is 

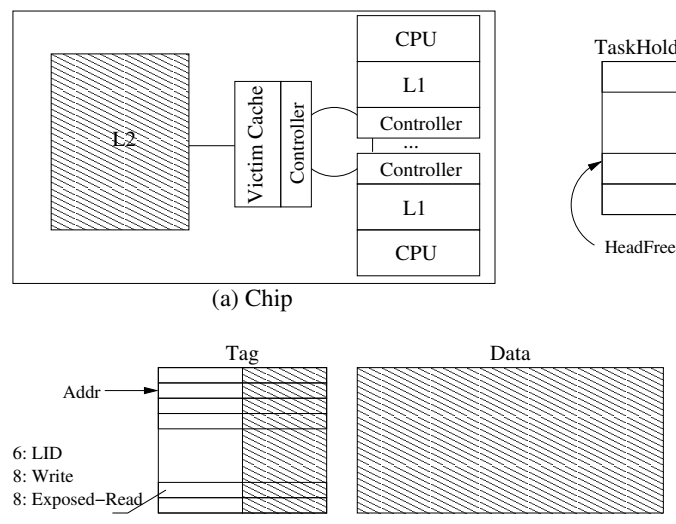

(c) L1 and Victim Cache Modifications
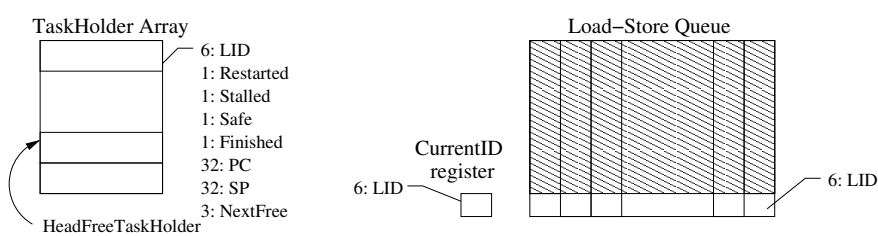

(b) Processor Modifications
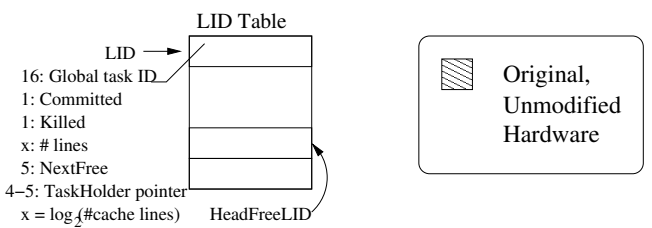

Figure 2: Proposed architecture of a high-performance TLS CMP.

kept in the CurrentID register of the load-store queue (Figure 2-(b)). This register is used to tag loads and stores as they are inserted in the load-store queue. With this support, when a load or store is sent to the L1, it includes the task's LID. Note that a processor can have in-flight instructions from multiple tasks.

In the L1s and the victim cache, each line tag is augmented with an LID and, for each word in the line, with one Write and one ExposedRead bit (Figure 2-(c)). As per Section 2, each cache keeps its own LID Table to translate LIDs to global task IDs (Figure 2-(c)). The LID Table is direct mapped. Each entry has information for one LID.

A novel feature of this architecture is that each LID Table entry also contains a Killed bit and a Committed bit for the corresponding task, and a counter of the number of lines in the cache with that LID. These fields are used to speed-up some of the tag-group operations of Section 3.2, as we will see in Section 4.2. Each entry also has a pointer to the corresponding TaskHolder.

The architecture does not include special hardware for register communication between cores. All dependences are enforced through memory. The reason is to minimize changes to off-the-shelf cores.

\subsection{Example: Use of the LID Table}

To show that our baseline TLS architecture is efficient, we give as an example the novel use of the LID Table for tag-group operations. Each LID Table entry is extended with summary-use information: the number of lines that the corresponding task still has in the cache, and whether the task has been killed or committed. With this extra information, all the tag-group operations of Section 3.2 are performed efficiently.

Specifically, when a task receives a squash or commit signal, its LID Table entry is updated by setting the Killed bit (Figure 3-(a)) or the Committed bit, respectively. No tag walking is performed.

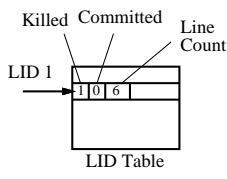

(a)
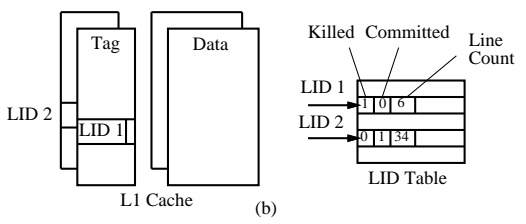

Figure 3: Using the LID Table on a task kill (a) and on a cache line replacement (b).

At any time, when a processor issues a load, if the load's address and the LID match one of the L1 tag entries, a hit occurs. In this case, the LID Table is not accessed. In all other cases, a miss occurs and the LID Table is accessed. We index the LID Table with the request's LID, obtain the corresponding global task ID, and include it in a request issued to the ring. Moreover, to decide which line to evict from the L1, we also index the LID Table with the LIDs of the lines that are currently using the cache set where space is needed (LID1 and LID2 in Figure 3-(b)). These are not time-critical accesses. If we find entries that have the Killed bit set (LID1 in the example), the count of cached lines is decremented, and the corresponding line in the cache is either chosen as the replacement victim or invalidated. Otherwise, if we find an entry with the Committed bit set (LID2 in the example), we use the line as the victim (possibly after a write back to memory), and decrement the count. If any one of these counters reaches zero, that LID is recycled.

\subsection{TLS Compiler}

We have built a TLS compiler [27] that adds several passes to a development branch of gcc 3.5. The branch uses a static single-assignment tree as the high-level intermediate representation [7]. With this approach, we leverage a complete compiler infrastructure. Our TLS passes generate tasks out of loop iterations and the code that follows (i.e., the continuation of) subroutines. The compiler first marks the tasks, and then tries to place spawn statements for each of these tasks as early in the code as it can. Only spawns that have moved up the code significantly are retained.

Before running the compiler, we run SGI's source-to-source optimizer (copt from MIPSPro), which performs PRE, loop unrolling, inlining, and other optimizations. As a result, the non-TLS code has a quality comparable to the MIPSPro SGI compiler for integer codes at O3. Code quality when TLS is enabled is not as good, as explained in Section 3.4.

The compilation process includes a simple profiler. The profiler takes the initial TLS executable, runs a tiny data set, and identifies those tasks that should be eliminated because they are unlikely to be of much benefit (Section 5.3.2). Then, the compiler re-generates the executable by eliminating these tasks.

\section{Energy-Saving TLS Optimizations}

To reduce the energy consumed by the TLS sources of Table 1, we can use many performance-oriented TLS optimizations proposed elsewhere. Examples are improvements to the cache hierarchy to minimize conflicts [5] or enhancements to the coherence protocol to reduce communication latency [23]. While these optimizations improve performance, they typically also reduce the energy consumed by a program.

In this paper, we are not interested in these optimizations. If they are cost-effective, they should already be included in any baseline TLS design. Instead, we are interested in energy-centric optimizations. These are optimizations that do not increase performance noticeably; in fact, they may even slightly reduce it. However, they reduce energy consumption significantly. They would not necessarily be included in a performance-centric TLS design. 
We propose three guidelines to identify energy-centric optimizations: (1) reduce the number of checks, (2) reduce the cost of individual checks, and (3) eliminate work with low performance returns. As examples, we propose simple, yet effective techniques. They are shown in the last column of Table 1.

\subsection{Reducing the Number of Checks}

\subsubsection{Avoid Eagerly "Walking" the Cache Tags in the Background (NoWalk)}

With the LID Table design described in Section 4.2, tag-group operations are very efficient. A task squash or commit only involves setting the Killed or Committed bit, respectively, in the LID Table. As lines belonging to squashed or committed tasks are eliminated from the cache due to replacements, the corresponding counts in the LID Table are decremented. When a count reaches zero, its associated LID can be recycled. Consequently, LID recycling is also very fast.

However, always waiting for LIDs to get "naturally" recycled in this way may hurt performance because we may run out of LIDs. Consequently, our baseline TLS architecture recycles LIDs in a more aggressive manner, inspired in previous work [17]. Specifically, it has a hardware FSM that periodically walks the tags of the cache in the background when the cache is idle. It uses the LID Table to identify tasks with the Killed bit set and then, when the cache is idle, it walks the cache tags to invalidate their lines and free up their LIDs. It also performs a similar operation to eagerly free up the LIDs of longcommitted tasks [18]. With this approach, performance is highest because we never run out of LIDs. However, we spend energy with many checks.

The energy optimization that we propose is to avoid in most cases any eager walk of the cache tags. Instead, we rely on the "natural" LID recycling when the associated count reaches zero, as described above. We only activate a background walk of the tags like in the baseline TLS architecture in one case: when there is only one free LID left. With this optimization, we occasionally may have to stall due to temporary lack of LIDs. However, we eliminate many tag checks.

\subsubsection{Lower Traffic to Check Version-IDs (TrafRed)}

In TLS, many messages are sent to check version IDs. For example, when a processor writes to a non-exclusive line, all the caches with a version of the requested line are typically checked, to see if there is an exposed read to the line from a more speculative task. Such task will be squashed. Similarly, on displacement of a committed line to L2, those same caches are checked, to invalidate older versions of the line. Such versions cannot remain cached anymore.

We propose a simple optimization to reduce the number of checks needed and, therefore, the traffic. Cache lines are extended with a Newest and an Oldest bit. Every time that a line is loaded into a cache, we set the Newest and/or Oldest bit if the line contains the latest and/or the earliest cached version, respectively, of the corresponding address. As execution proceeds, Newest may be reset on an access by another task. With this support, if a processor writes on a Newest line cached locally, there is no need to check other caches for exposed reads. Similarly, if a processor displaces a committed line with the Oldest bit set, there is no need to check other caches for older versions. This optimization applies to two rows in Table 1.

\subsection{Reducing the Cost of Individual Checks}

A simple example is to tag cache lines with short LIDs rather than global task IDs. This approach of using indirection is well known [22]. Consequently, we already use it in the baseline TLS CMP and do not evaluate its impact. We call it Indirect in Table 1.

\subsection{Eliminating Low-Return Work}

\subsubsection{Stall a Task After Two Squashes (StallSq)}

A simple technique is to limit the number of times that a task is allowed to restart after a squash. After a task has been squashed $N$ times, it is not given a CPU again until it becomes non-speculative.

We performed experiments always restarting tasks immediately after they are squashed. We found that $73.0 \%$ of the tasks are never squashed, $20.6 \%$ are squashed once, $4.1 \%$ twice, $1.4 \%$ three times, and $0.9 \%$ four times or more. Restarting a task after its first squash can be beneficial, as the L2 cache and branch predictor have been warmed up. Restarting after further squashes delivers low performance returns while steadily consuming more energy. Consequently, we reset and stall a task after its second squash. This is accomplished with two bits per TaskHolder entry (Figure 2): the Restarted bit is set after the task has been squashed and restarted once; the Stalled bit is set after the second squash.

\subsubsection{Energy-Aware Task Pruning (TaskOpt)}

The profiler in our compilation pass includes a simple model that identifies tasks that should be eliminated because they are unlikely to be beneficial. The main focus is on tasks that cause squashes. For the baseline TLS architecture, the model minimizes the duration of the program. The energy-centric optimization is to use a model that minimizes the product Energy $\times$ Delay $^{2}$ for the program.

Our compiler generates a binary with task spawn instructions (Figure 4-(a)). The profiler runs the binary sequentially, using the Train data set for SPECint codes. As the profiler executes a task, it records the variables written. When it executes tasks that would be spawned earlier, it compares the addresses read against those written by predecessor tasks. With this, it can detect potential run-time violations. The profiler also models a simple cache to estimate the number of misses in the machine's L2. For performance, cache timing is not modeled. On average, the profiler takes around 5 minutes to run on a $3 \mathrm{GHz}$ Pentium 4.

The profiler estimates if a task squash will occur and, if so, the number of instructions squashed $I_{\text {squashed }}$ (Figure 4-(b)) and the final instruction overlap after re-execution $I_{\text {overlap }}$ (Figure 4-(c)). In addition, the profiler estimates the number of L2 misses $M_{\text {squashed }}$ in the squashed instructions. These misses will have a prefetching effect that will speed up the re-execution of $T 2$.

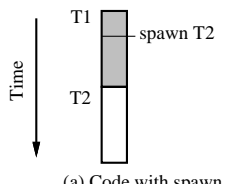

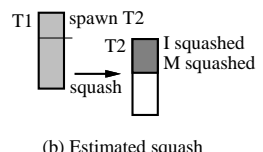

(b) Estimated squash

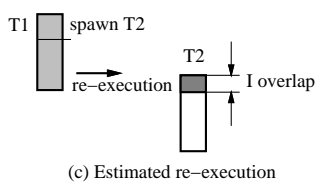

(c) Estimated re-execution
Figure 4: Modeling task squash and restart. T1 and T2 are tasks.

Assuming that each instruction takes $T_{i}$ cycles to execute, and an L2 miss stalls the processor for $T_{m}$ cycles, the estimated execution time reduction $\left(T_{\text {red }}\right)$ is $I_{\text {overlap }} \times T_{i}+M_{\text {squashed }} \times T_{m}$. Assuming that the energy consumed by each instruction is $E_{i}$, the approximate increase in energy $\left(E_{\text {inc }}\right)$ is $I_{\text {squashed }} \times E_{i}$.

Our profiler focuses on tasks that have a rate of squashes per commit higher than $R_{\text {squash }}$. In the baseline architecture, it eliminates a task if $T_{r e d}$ is less than a threshold $T_{p e r f}$. In our our energyoptimized architecture, it eliminates a task if subtracting $T_{\text {red }}$ from the program time and adding $E_{i n c}$ to the program energy, the program's $E \times D^{2}$ product increases. In this case, voltage-frequency scaling could (ideally) do better.

The values of the thresholds and parameters used are listed in Table 2. This optimization has significant impact: on average, the profiler eliminates $39.9 \%$ of the static tasks in performance mode, and $49.2 \%$ in energy mode. 


\begin{tabular}{|c|c|c|c|c|c|}
\hline \multicolumn{6}{|c|}{ TLS CMP with four 3-issue cores (TLS4-3i) } \\
\hline \multicolumn{6}{|c|}{ Processor } \\
\hline \multirow{7}{*}{\multicolumn{3}{|c|}{$\begin{array}{l}\text { Frequency: 5.0 GHz @ } 70 \mathrm{~nm} \\
\text { Branch penalty: } 13 \text { cyc (min) } \\
\text { RAS: } 32 \text { entries } \\
\text { BTB: } 2 \mathrm{~K} \text { entries, } 2 \text {-way assoc. } \\
\text { Branch predictor (spec. update): } \\
\text { bimodal size: } 16 \mathrm{~K} \text { entries } \\
\text { gshare-11 size: } 16 \mathrm{~K} \text { entries }\end{array}$}} & \multicolumn{3}{|c|}{ Fetch/issue/comm width: $6 / \mathbf{3} / 3$} \\
\hline & & & & \multicolumn{2}{|l|}{ I-window/ROB size: $68 / 126$} \\
\hline & & & & \multicolumn{2}{|l|}{ Int/FP registers: 90/68 } \\
\hline & & & & \multicolumn{2}{|l|}{ LdSt/Int/FP units: $1 / 2 / 1$} \\
\hline & & & & \multicolumn{2}{|l|}{ Ld/St queue entries: $48 / 42$} \\
\hline & & & & TaskHolders/processor: 8 & \\
\hline & & & & \multicolumn{2}{|c|}{ TaskHolder access time, energy: 1 cyc, $0.25 n$} \\
\hline Cache & D-L1 & $\mathrm{VC}$ & L2 & D-L & $\mathrm{VC}$ \\
\hline Size: & $16 \mathrm{~KB}$ & $4 \mathrm{~KB}$ & $1 \mathrm{MB}$ & LID Table: & \\
\hline RT: & 3 cyc & 8 сус & 10 cyc & entries/ports: $\quad 64 / 2$ & $32 / 1$ \\
\hline Assoc: & 4-way & 4-way & 8-way & acc time/energy: 1 cyc/0.11n & $1 \mathrm{cyc} / 0.07 \mathrm{~nJ}$ \\
\hline Line size: & $64 \mathrm{~B}$ & $64 \mathrm{~B}$ & $64 \mathrm{~B}$ & & \\
\hline Ports: & 1 & 1 & 1 & Latency from spawn to new th & $\mathrm{d}: 14$ cyc \\
\hline Pend ld/st: & 16 & 64 & 64 & & \\
\hline
\end{tabular}

\begin{tabular}{|c|c|c|c|}
\hline \multicolumn{4}{|c|}{ 6-issue superscalar chip (Uni-6i) } \\
\hline \multicolumn{4}{|c|}{ Processor } \\
\hline \multicolumn{3}{|c|}{$\begin{array}{l}\text { Frequency: 5.0 GHz @ } 70 \mathrm{~nm} \\
\text { Branch penalty: } 13 \text { cyc (min) } \\
\text { RAS: } 32 \text { entries } \\
\text { BTB: } 2 \mathrm{~K} \text { entries, } 2 \text {-way assoc. } \\
\text { Branch predictor (spec. update): } \\
\quad \text { bimodal size: } 16 \mathrm{~K} \text { entries } \\
\quad \text { gshare-11 size: } 16 \mathrm{~K} \text { entries }\end{array}$} & $\begin{array}{l}\text { Fetch/issue/comm width: } 6 / \mathbf{6} / 6 \\
\text { I-window/ROB size: } 104 / 204 \\
\text { Int/FP registers: } 132 / 104 \\
\text { LdSt/Int/FP units: } 2 / 4 / 2 \\
\text { Ld/St queue entries: } 66 / 54\end{array}$ \\
\hline Cache & D-L1 & L2 & \\
\hline Size: & $16 \mathrm{~KB}$ & $1 \mathrm{MB}$ & \\
\hline RT: & 2 cyc & 10 cyc & \\
\hline Assoc: & 4-way & 8-way & \\
\hline Line size: & $64 B$ & $64 \mathrm{~B}$ & \\
\hline Ports: & 2 & 1 & \\
\hline Pend ld/st: & 16 & 64 & \\
\hline
\end{tabular}

\begin{tabular}{||l|l||}
\hline \hline I-L1 & Size: 16KB; RT: 2 cyc; assoc: 2-way; line size: 64B; ports: 1 \\
Bus \& Memory & FSB frequency: 533MHz; FSB width: 128bit; memory: DDR-2; DRAM bandwidth: $8.528 \mathrm{~GB} / \mathrm{s} ;$ memory RT: $98 \mathrm{~ns}$ \\
Profiling parameters & $R_{\text {squash }}: 0.8 ; T_{i}: 1 ; T_{m}: 200$ cyc; $E_{i}: 8 \mathrm{pJ} ; T_{\text {perf }}: 90$ cyc; Size $_{\text {energy }}: 45 ;$ Size $_{\text {perf }}: 30 ;$ Hoist $_{\text {energy }}: 120 ;$ Hoist $_{\text {perf }}: 110$ \\
\hline \hline
\end{tabular}

Table 2: TLS CMP with four 3-issue cores (TLS4-3i) and 6-issue superscalar chip (Uni-6i) modeled. In the table, RAS, FSB, RT, and VC stand for Return Address Stack, Front-Side Bus, minimum Round-Trip time from the processor, and Victim Cache, respectively. Cycle counts refer to processor cycles.

\subsubsection{Eliminate Low-Return Tasks (TaskOpt)}

Another energy-centric optimization is for the compilation pass to aggressively remove tasks whose size is small or whose spawn point has not been moved up in the code much. We use a threshold size Size energy and threshold spawn hoist distance Hoist energy $_{\text {en }}$ that are more aggressive than their performance-centric counterparts

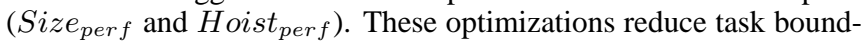
aries and code bloat. They eliminate $36.1 \%$ of the static tasks in energy mode compared to $34.7 \%$ in performance mode. For ease of presentation, we combine this technique and the previous one into TaskOpt in our evaluation, since they are very related.

\subsection{Summary}

We place the optimizations in the corresponding row of Table 1. As indicated in Section 5.2, Indirect is not evaluated.

\section{Evaluation Setup}

To assess the energy efficiency of TLS, we compare an TLS CMP to a non-TLS chip that has a single processor of the same or wider issue width. We use execution-driven simulations, with detailed models of out-of-order superscalars and advanced memory hierarchies, enhanced with models of dynamic and leakage energy from Wattch [3], Orion [28], and HotLeakage [29].

\subsection{Architectures Evaluated}

The TLS CMP that we propose has four 3-issue cores, the microarchitecture of Section 4, and the energy-centric TLS optimizations of Section 5. We call the chip TLS4-3i. The non-TLS chips have a single superscalar with a conventional L1 and L2 on-chip cache hierarchy. We consider two: one is a 6-issue superscalar (Uni-6i) and the other a 3 -issue superscalar (Uni-3i). We choose to compare the TLS4-3i and Uni-6i designs because both chips have approximately the same area, as can be estimated from $[11,20]$.

Table 2 shows the parameters for TLS4-3i and Uni-6i. As we move from 3-issue to 6-issue cores, we scale all the processor structures (e.g., ports, FUs, etc) according to the issue width of the core. We try to create a balanced processor as much as possible, by scaling the processor resources. This is the same approach used in IBM's Power 4.

In our comparison, we favor Uni-6i. We assume that Uni-6i has the same frequency and the same pipeline depth as the cores in TLS4$3 i$. This helps Uni-6i because, in practice, a 6-issue core would not cycle as fast as a 3-issue core with the same pipeline. For example, according to CACTI [20], the access time of the register file and the instruction window in Uni-6i would be at least 1.25 times higher and 1.35 times higher, respectively, than in the TLS4-3i cores. Moreover, extrapolating results from [15], the bypass network would have 2.6 times longer latency in Uni-6i (assuming a fully-connected bypass network). In our simulations, however, we assume the same frequency for Uni-6i and TLS4-3i.

Since both processors have the same pipeline depth and branch misprediction penalty, we feel that it is fair to also give them the same branch predictor. In addition, both processors have an integer and an FP cluster. Since we run integer codes in the evaluation, the FP cluster is clock-gated almost all the time.

The tag array in TLS4-3i's L1 caches is extended with the LID, and the Write and Exposed-Read bits (Figure 2). At worst, the presence of these bits increases the access time of the L1 only slightly. To see why, note that the LID bits can simply be considered part of the line address tag, as a hit requires address and LID match. Moreover, in our protocol, the Write and Exposed-Read bits are not checked before providing the data to the processor; they may be updated after that. However, to be conservative, we increase the L1 access latency in TLS4-3i one cycle over Uni-6i, to 3 cycles.

$U n i-3 i$ is like $U n i-6 i$ except that the core is 3-issue, like those in $T L S 4-3 i$, and the $\mathrm{L} 1$ cache only has 1 port. For completeness, we also evaluate one additional chip: TLS2-3i. TLS2-3i is a TLS CMP like $T L S 4-3 i$, but with only two cores.

To maximize the use of commodity hardware, the TLS CMP has no special hardware support for inter-processor register communication. $[18,19]$ have more details on the architecture evaluated.

\subsection{Energy Considerations}

We estimate and aggregate the dynamic and leakage energy consumed in all chip structures, including processors, cache hierarchies, and on-chip interconnect. For the dynamic energy, we use the Wattch [3] and Orion [28] models. We apply aggressive clock gating to processor structures in all cores. In addition, unused cores in the TLS CMP are also clock gated. Activating and deactivating corewide clock gating takes 100 cycles each time. Clock-gated structures are set to consume $5 \%$ of their original dynamic energy, which is one of the options in Wattch. We extend the Wattch models to support our deeper pipelines and to take into account the area when computing the clock energy. The chip area is estimated using data from [11] and CACTI [20].

Leakage energy is estimated with HotLeakage [29], which models both sub-threshold and gated leakage currents. We use an iterative ap- 


\begin{tabular}{|c|c|c|c|c|c|c|c|c|c|c|c|c|c|c|c|c|}
\hline \multirow[t]{2}{*}{ Apps } & \multicolumn{3}{|c|}{$\begin{array}{l}\text { Squashed } \\
\text { Instructions } \\
(\%)\end{array}$} & \multicolumn{3}{|c|}{$\begin{array}{l}\text { Busy } \\
\text { CPUs }\end{array}$} & \multirow{2}{*}{$\begin{array}{c}\text { Pruned } \\
\text { Tasks } \\
(\%) \\
\text { Task } \\
\text { Opt } \\
\end{array}$} & \multicolumn{2}{|c|}{$\begin{array}{c}\text { Task } \\
\text { Size } \\
\text { (Instructions) } \\
\end{array}$} & \multirow{2}{*}{$\begin{array}{c}E D^{2} \\
\text { Reduc. } \\
(\%) \\
\text { Task } \\
\text { Opt }\end{array}$} & \multicolumn{2}{|c|}{$\begin{array}{l}\text { Ratio of Tag } \\
\text { Accesses } \\
\text { (TLS/Uni-3i) }\end{array}$} & \multicolumn{2}{|c|}{$\begin{array}{l}\text { Traffic } \\
\text { (TLS/ } \\
\text { Uni-3i) }\end{array}$} & \multicolumn{2}{|c|}{$\begin{array}{l}\text { Add'l Instruct. } \\
\text { in Non-Squashed } \\
\text { Dyn. Tasks }(\%)\end{array}$} \\
\hline & $\begin{array}{l}\text { No } \\
\text { Opt }\end{array}$ & $\begin{array}{c}\text { Stall } \\
\text { Sq }\end{array}$ & $\begin{array}{l}\text { Task } \\
\text { Opt }\end{array}$ & $\begin{array}{l}\text { No } \\
\text { Opt } \\
\end{array}$ & $\begin{array}{c}\text { Stall } \\
\mathrm{Sq} \\
\end{array}$ & $\begin{array}{l}\text { Task } \\
\text { Opt }\end{array}$ & & $\begin{array}{l}\text { No } \\
\text { Opt } \\
\end{array}$ & $\begin{array}{c}\text { Task } \\
\text { Opt } \\
\end{array}$ & & $\begin{array}{l}\text { No } \\
\text { Opt }\end{array}$ & $\begin{array}{c}\text { No } \\
\text { Walk } \\
\end{array}$ & $\begin{array}{l}\text { No } \\
\text { Opt } \\
\end{array}$ & $\begin{array}{l}\text { Traf } \\
\text { Red } \\
\end{array}$ & $\begin{array}{l}\text { No } \\
\text { Opt }\end{array}$ & $\begin{array}{c}\text { Stall } \\
\mathrm{Sq}\end{array}$ \\
\hline bzip2 & 9.9 & 7.5 & 9.9 & 1.40 & 1.35 & $\overline{1.41}$ & 21.8 & 743.4 & 751.7 & -0.3 & 3.2 & 1.3 & 14.0 & 2.5 & 5.6 & 5.6 \\
\hline crafty & 26.2 & 25.4 & 18.9 & 1.97 & 1.95 & 1.70 & 6.2 & 932.0 & 1064.0 & 6.8 & 2.9 & 2.0 & 7.1 & 3.6 & 5.6 & 5.6 \\
\hline gap & 35.2 & 31.6 & 35.1 & 2.07 & 1.94 & 2.06 & 14.6 & 1270.3 & 1280.4 & -0.8 & 3.6 & 2.2 & 16.7 & 8.4 & 3.8 & 3.8 \\
\hline gzip & 14.0 & 14.0 & 11.9 & 1.49 & 1.48 & 1.49 & 7.5 & 626.6 & 634.2 & 0.3 & 3.5 & 1.9 & 12.3 & 4.0 & 6.5 & 6.5 \\
\hline $\mathrm{mcf}$ & 28.8 & 28.7 & 28.8 & 2.38 & 2.38 & 2.38 & 0.4 & 47.9 & 47.9 & 0.0 & 3.8 & 2.7 & 42.0 & 11.5 & 31.9 & 31.9 \\
\hline parser & 39.3 & 29.9 & 13.8 & 2.03 & 1.85 & 1.25 & 18.9 & 167.3 & 261.6 & 26.4 & 3.6 & 3.2 & 9.8 & 7.1 & 20.8 & 18.0 \\
\hline twolf & 4.4 & 4.4 & 4.4 & 1.62 & 1.62 & 1.62 & 0.4 & 409.4 & 409.4 & 0.0 & 3.3 & 1.6 & 55.9 & 3.2 & 6.5 & 6.5 \\
\hline vortex & 15.7 & 15.4 & 7.7 & 1.82 & 1.81 & 1.49 & 9.2 & 488.3 & 881.5 & 16.0 & 2.9 & 1.9 & 7.4 & 3.9 & 7.5 & 7.4 \\
\hline vpr & 29.5 & 29.2 & 27.9 & 3.14 & 3.13 & 2.61 & 17.0 & 212.9 & 389.1 & 10.4 & 3.2 & 3.1 & 10.9 & 6.4 & 23.9 & 21.2 \\
\hline Avg & 22.6 & 20.7 & 17.6 & 2.00 & 1.95 & 1.78 & 10.7 & 544.2 & 635.5 & 6.5 & 3.3 & 2.2 & 19.6 & 5.6 & 12.5 & 11.9 \\
\hline
\end{tabular}

Table 3: Architectural characteristics of the 4-core TLS CMP related to sources of energy consumption and their optimization.

proach suggested by Su et al. [24]: the temperature is estimated based on the current total power, the leakage power is estimated based on the current temperature, and the leakage power is added to the total power. This is continued until convergence. The maximum temperature at the junction for any application is not allowed to go beyond $85^{\circ} \mathrm{C}$, as recommended by the SIA Roadmap [1].

From our calculations, the average power consumed by the Uni$3 i$ and Uni-6i chips for the SPECint 2000 applications is 32 and 60 $\mathrm{W}$, respectively (more data will be shown later). Of this power, leakage accounts for $38 \%$ and $32 \%$, respectively. The majority of the dynamic power increase from Uni-3i to Uni-6i is due to five structures that more than double their contribution, largely because they double the number of ports. These are the rename table, register file, I-window, L1 data cache, and data TLB. In addition, the data forwarding network also increases its dynamic contribution by $70 \%$.

\subsection{Applications Evaluated}

We measure full SPECint 2000 applications with the Reference data set except eon, which is in $\mathrm{C}++$, and $g c c$ and perlbmk, which our compiler infrastructure does not compile. By full applications, we mean that we include all the code in the measurement, not just the more parallel sections such as loops. Uni-3i and Uni-6i run the binaries compiled with our TLS passes disabled. Such binaries have a code quality comparable to integer codes generated by the MIPSPro SGI compiler with $O 3$ (Section 4.3).

TLS and non-TLS binaries are very different. Therefore, we cannot compare the execution of a fixed number of instructions. Instead, we insert "simulation markers" in the code and simulate for a given number of markers. After skipping the initialization (several billion instructions), we execute up to a certain number of markers so that Uni-6i graduates from 750 million to 1.5 billion instructions.

\section{Evaluation}

In our evaluation, we first characterize the TLS CMP architecturally, with and without the energy optimizations. Then, we examine the energy cost of TLS and the savings of the optimizations. Finally, we compare the energy, power, and performance of the different chips. In the following, NoOpt is TLS4-3i without the optimizations.

\subsection{Architectural Characterization of the TLS CMP}

We measure architectural characteristics of the 4-core TLS CMP that are related to Table 1's sources of TLS energy consumption and optimizations. The data are shown in Table 3. In the table, we compare the chip before optimization (NoOpt), to the chip with one optimization at a time (StallSq, TaskOpt, NoWalk, or TrafRed).

The first TLS source of energy consumption in Table 1 is task squashing. Column 2 of Table 3 shows that, on average, NoOpt loses to task squashes $22.6 \%$ of the dynamic instructions executed. This is a significant waste. With our optimizations, we reduce the number of such instructions. Specifically, the average fraction becomes $20.7 \%$ with StallSq (Column 3) and $17.6 \%$ with TaskOpt (Column 4). Although not shown in the table, the fraction becomes $16.9 \%$ with both optimizations combined.

The next few columns of Table 3 provide more information on the impact of StallSq and TaskOpt. Under NoOpt, the average number of busy CPUs is 2.00 (Column 5). Since StallSq stalls tasks that are likely to be squashed and TaskOpt removes them, they both reduce CPU utilization. Specifically, the average number of busy CPUs is 1.95 and 1.78 with StallSq and TaskOpt, respectively (Columns 6 and 7). With both optimizations, the average can be shown to be 1.75 .

TaskOpt has a significant impact on the tasks. Recall from Sections 5.3.2 and 5.3.3 that, on average, NoOpt already prunes $74.6 \%$ of the static tasks using performance-only models. On top of that, TaskOpt prunes an additional $10.7 \%$ of the static tasks (Column 8). As a result, TaskOpt increases the average task size from 544 instructions in NoOpt (Column 9) to 635 (Column 10). Moreover, the average $E \times D^{2}$ of the applications, a metric for time and energy efficiency of computation [14], decreases by $6.5 \%$ (Column 11).

The second TLS source of energy in Table 1 is dominated by accesses to L1 cache tags. Such accesses in TLS are both more expensive (since tags have version IDs) and more frequent (e.g., due to tag-group operations). Column 12 of Table 3 shows that, on average, NoOpt has 3.3 times the number of tag checks in Uni-3i. However, with our NoWalk optimization, we eliminate many of these checks. Specifically, Column 13 shows that, with NoWalk, TLS only has 2.2 times as many tag checks as Uni-3i. Note that these figures include the contribution of squashed tasks.

The third TLS source of energy is additional traffic. Column 14 of Table 3 shows that, on average, NoOpt has 19.6 times the traffic of Uni-3i. To compute the traffic, we add up all the bytes of data or control passed between caches. This traffic increase is caused by the factors described in Section 3.3. However, after we apply our TrafRed optimization, the traffic reduces considerably. On average, with TrafRed, TLS only has 5.6 times the traffic of Uni-3i (Column 15).

The fourth TLS source of energy is additional instructions. Column 16 shows that NoOpt executes on average $12.5 \%$ more dynamic instructions in non-squashed tasks than Uni-3i. The TaskOpt optimization, by eliminating small and inefficient tasks, reduces the additional instructions to $11.9 \%$ on average (Column 17).

\subsection{The Energy Cost of TLS ( $\left.\Delta E_{T L S}\right)$}

In Section 3, we defined the energy cost of TLS $\left(\Delta E_{T L S}\right)$ as the difference between the energy consumed by our TLS CMPs and Uni$3 i$. Figure 5 characterizes $\Delta E_{T L S}$ for our 4-core TLS CMP. The figure shows six bars for each application. They correspond to the total energy consumed by the chip without any optimization (NoOpt), with individual optimizations enabled (StallSq, TaskOpt, NoWalk, and TrafRed), and with all optimizations applied (TLS4-3i). For each application, the bars are normalized to the energy consumed by Uni- 


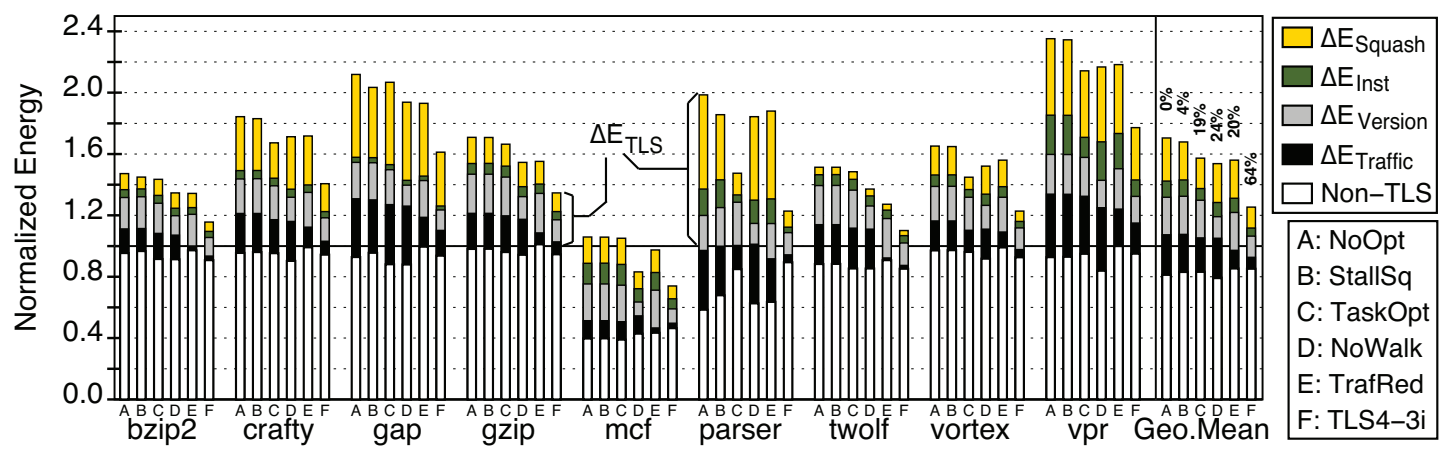

Figure 5: Energy cost of TLS $\left(\Delta E_{T L S}\right)$ for our 4-core TLS CMP chip with and without energy-centric optimizations. The percentages listed above the average bars are the decrease in $\Delta E_{T L S}$ when the optimizations are enabled.

3i. Consequently, the difference between the top of the bars and 1.00 is $\Delta E_{T L S}$.

Each bar in Figure 5 is broken into the contributions of the TLSspecific sources of energy consumption listed in Table 1 . These include task squashing $\left(\Delta E_{\text {Squash }}\right)$, additional dynamic instructions in non-squashed tasks $\left(\Delta E_{I n s t}\right)$, hardware for data versioning and dependence checking $\left(\Delta E_{\text {Version }}\right)$, and additional traffic $\left(\Delta E_{\text {Traffic }}\right)$. The rest of the bar (Non-TLS) is energy that we do not attribute to TLS.

Ideally, $\Delta E_{T L S}$ should be roughly equal to the addition of the four TLS-specific sources of energy consumption and, therefore, Non-TLS should equal 1 . In practice, this is not the case because a given program runs on the TLS CMP and on Uni-3i at different speeds and temperatures. As a result, the "non-TLS" dynamic and leakage energy varies across runs, causing Non-TLS to deviate from 1 . In fact, since for all applications the TLS CMP is faster than Uni-3i (i.e., the TLS bars in Figure 1-(a) are over 1), Non-TLS is less than 1: nonTLS hardware structures have less time to leak or to spend dynamic energy cycling idly.

If we consider the NoOpt bars, we see that the energy cost of unoptimized TLS ( $\left.\triangle E_{T L S}\right)$ is significant. On average, unoptimized TLS adds $70.4 \%$ to the energy consumed by Uni-3i. We also see that all four of our TLS sources of energy consumption contribute noticeably. Of them, task squashing consumes the most energy, while additional instructions consumes the least.

\subsection{The Impact of Energy-Centric Optimizations}

The rest of the bars in Figure 5 show the impact of our energy-centric optimizations on the TLS energy sources. From the figure, we see that each optimization effectively reduces the TLS energy sources that it is expected to minimize from Table 1. This is best seen from the average bars.

Consider TaskOpt first. In Figure 5, TaskOpt reduces $\Delta E_{\text {Squash }}$ and $\Delta E_{\text {Inst }}$ - its targets in Table 1 . This is consistent with Table 3, where TaskOpt reduces the fraction of squashed instructions from $22.6 \%$ to $17.6 \%$, and decreases the additional dynamic instructions in non-squashed tasks from $12.5 \%$ to $11.9 \%$.

Consider now NoWalk. In Figure 5, NoWalk mostly reduces $\Delta E_{V e r s i o n}-$ its target in Table 1 . This was expected from Table 3, where NoWalk reduces the number of tag accesses relative to Uni-3i from 3.3 times to 2.2 times. In addition, since it reduces the temperature, it also reduces the leakage component in Non-TLS slightly.

If we consider TrafRed in Figure 5, we see that it mostly reduces $\Delta E_{\text {Traffic }}-$ its target in Table 1. Again, this is consistent with Table 3, where TrafRed reduces the traffic relative to Uni-3i from 19.6 times to 5.6 times on average.

Finally, StallSq only addresses $\Delta E_{S q u a s h}$, which is its target in Table 1. As expected from the modest numbers in Table 3, where it reduces squashed instructions from $22.6 \%$ to $20.7 \%$, it has a small impact in Figure 5.

This analysis shows that each of TaskOpt, NoWalk, and TrafRed effectively reduces a different energy source, and that the three techniques combined cover all sources considered. Consequently, when we combine all four optimizations in TLS4-3i, all TLS sources of consumption decrease substantially. The resulting $T L S 4-3 i$ bar shows the true energy cost of TLS. If we measure the section of the bar over 1.00 , we see that this cost is on average only $25.4 \%$. We feel that this is a remarkably low energy overhead for TLS.

With our simple optimizations, we have been able to eliminate on average $64 \%$ of $\Delta E_{T L S}$. Compared to the overall on-chip energy consumed by $\mathrm{NoOpt}$, this is a very respectable energy reduction of $26.5 \%$. Moreover, as we will see later, the applications have only been slowed down on average by less than $2 \%$.

Finally, an analysis of individual applications reveals many interesting facts. Unfortunately, space limitations prevent any deep discussion. We only note that $m c f$ has a negative $\Delta E_{T L S}$ in some cases. The reason is that, without TLS, the L2 suffers frequent misses; with TLS, tasks prefetch data for other tasks, removing misses and speeding up the execution significantly (Section 7.5). The result is that the TLS CMP has less time to leak and to spend dynamic energy cycling, hence Non-TLS is very small.

\subsection{Comparing Energy Consumption Across Chips}

Figure 6 compares the energy consumed by our optimized TLS4-3i chip and Uni-3i, Uni-6i and, for completeness, TLS2-3i. Each bar is normalized to Uni-3i and broken down into dynamic energy consumed by the clock, core, and memory subsystem, and leakage energy. The memory category includes caches, TLBs, and interconnect.

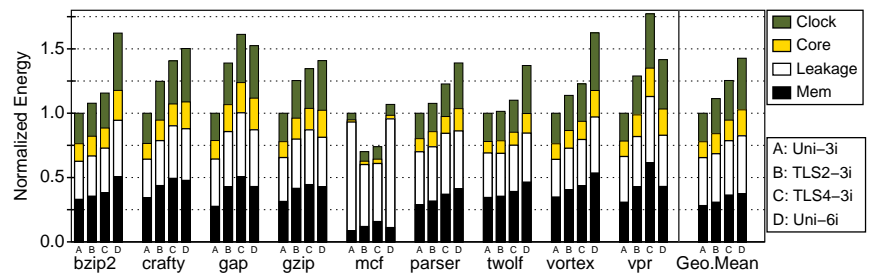

Figure 6: Comparing the energy consumption after TLS CMP optimization. All bars are normalized to Uni-3i.

Consider first Uni-6i. Its core, clock, and memory categories are larger than in Uni-3i because of the bigger structures in the wide processor. Specifically, the rename table, register file, I-window, L1 data cache, and data TLB have twice the number of ports. This roughly doubles the energy per access [20]. Furthermore, all these structures but the cache and TLB also have more entries. Finally, the forwarding network also increases its complexity and, therefore, its consumption. The figure also shows that leakage has increased. The reason is that, while Uni-6i is faster than $U n i-3 i$, it consumes the highest average power (Section 7.5) and, therefore, has a higher temperature. Temperature has an exponential impact on leakage.

Compared to Uni-6i, TLS4-3i has smaller core and clock energies 


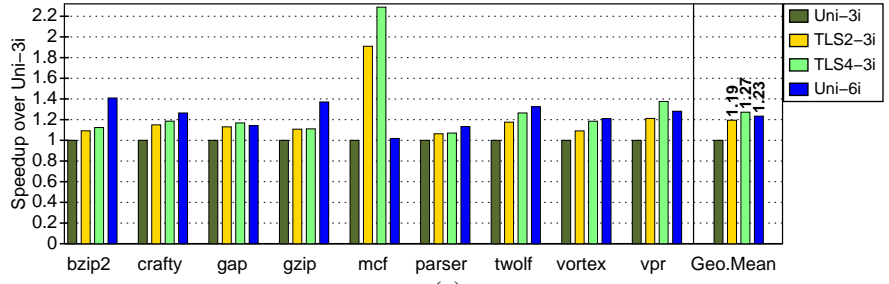

(a)

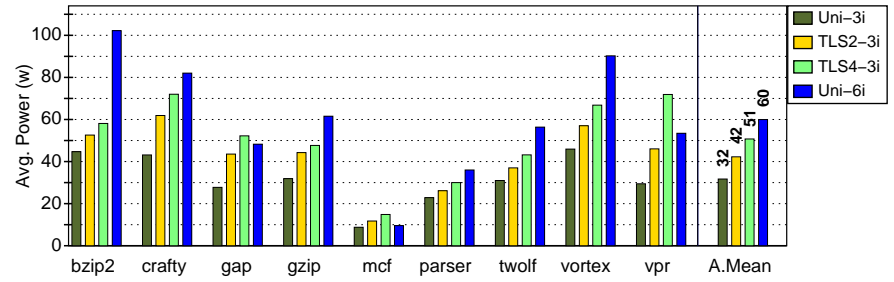

(b)

Figure 7: Execution speedup relative to Uni-3i (a), and average power consumption (b) for different chips. Note that the mean used for speedups is the geometric one.

because it has the simpler hardware structures of Uni-3i. Its leakage is also smaller because its average power (Section 7.5) and, therefore, temperature are lower than Uni-6i. Its memory category, however, is slightly higher than Uni-6i. The reason is TLS4-3i's higher consumption in structures and traffic to support data versioning and dependence checking.

\subsection{Comparing Performance/Power Across Chips}

Finally, we take the optimized TLS4-3i and compare its performance, and average power to other chips. Figure 7-(a) shows application speedups relative to execution on Uni-3i, while Figure 7-(b) shows the average power consumed during execution. As usual, $T L S 2-3 i$ is also shown. As a reference, the arithmetic mean of the average IPC of the applications on TLS4-3i is 1.38 .

Figure 7-(a) shows that, on average, TLS4-3i delivers a speedup of 1.27 over Uni-3i. This shows that our TLS compiler successfully extracts good tasks from these irregular codes. This speedup is slightly lower than the 1.29 speedup shown in Figure 1-(a). The reason is that our energy-centric optimizations reduce performance slightly.

Figure 7-(a) also shows that TLS4-3i is on average faster than Uni$6 i$. The speculative parallelism enabled by TLS4-3i in these hardto-parallelize codes is more effective than doubling the issue width. This is a good result, especially because it conservatively assumes the same frequency for both chips. In practice, designing the wider issue processor at this high frequency is likely to be more challenging.

Note that while the TLS4-3i speedup for most codes ranges from 1.10 to $1.35, m c f$ exhibits a higher speedup. As indicated in Section 7.3, $m c f$ benefits from constructive data prefetching into L2 by TLS tasks. Without considering $m c f$, the geometric mean of TLS4$3 i$ 's speedup is 1.18 , which is still comparable to Uni-6i's, always assuming the same frequency.

On the other hand, Figure 7-(b) shows that the average on-chip power consumed by TLS4-3i is typically lower than Uni-6i's. On average, it is $15 \%$ lower. Moreover, it never reaches the high values that Uni-6i dissipates in some applications. If we compare Figure 7(b) to Figure 1-(b), we see the effectiveness of our optimizations at reducing the power consumed by the four-core TLS CMP.

We also compare the average $E \times D^{2}$ of TLS4-3i and Uni-6i. Unfortunately, due to lack of space, we cannot show the complete set of data. On average, TLS4-3i's $E \times D^{2}$ is $7.6 \%$ lower than Uni-6i's. We conclude, therefore, that TLS4-3i is more energy-efficient than Uni-6i.

We can get further insight if we analytically apply ideal voltagefrequency scaling. We assume that performance is linearly proportional to frequency and scale frequency and voltage proportionally. We also assume that average dynamic power is proportional to the cube of frequency and that average leakage power is linearly proportional to voltage [4]. Then, for each chip, we can derive a curve that relates the average power consumption with performance as:

$P_{\text {new }}^{\text {total }}=P_{\text {orig }}^{\text {dyn }} \times\left(\frac{\text { Speedup }_{\text {new }}}{\text { Speedup }_{\text {orig }}}\right)^{3}+P_{\text {orig }}^{\text {leak }} \times\left(\frac{\text { Speedup }_{\text {new }}}{\text { Speedup }_{\text {orig }}}\right)$

Figure 8 shows the resulting curves for TLS4-3i and Uni-6i. Each curve follows possible speedup-power working points for one chip. The lower a curve is, the more energy-efficient the architecture is. Each curve shows one data point, which corresponds to the actual working conditions in our experiments.

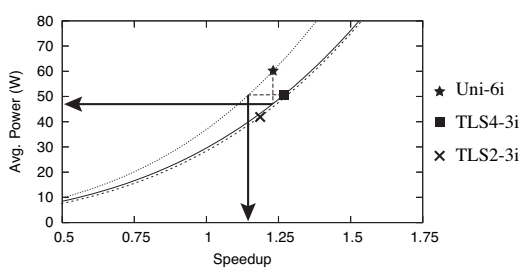

Figure 8: Ideal relation between speedup and average power.

We can see that Uni-6i is less energy-efficient than TLS4-3i. If we scale down TLS4-3i's frequency until TLS4-3i's performance is equal to Uni-6i's, TLS4-3i consumes $20 \%$ less power than Uni-6i (horizontal arrow). Alternatively, if we scale down Uni-6i's frequency until Uni-6i's power is equal to TLS4-3i's, TLS4-3i is $13 \%$ faster than Uni$6 i$ (vertical arrow).

Finally, Figure 8 also shows a curve for $T L S 2-3 i$. The data shows that $T L S 2-3 i$ has a very small efficiency advantage over $T L S 4-3 i$.

\subsection{Summary}

The fundamental reason why TLS CMPs can be more energyefficient than wider-issue superscalars is that energy scales superlinearly and performance sublinearly with the size of processor structures. Consequently, multiple simple TLS cores can be more efficient than a single wide core, as long as (i) TLS's hardware overheads and (ii) TLS's wasted work are kept to a minimum.

We have addressed these issues with a lean TLS CMP microarchitecture and a set of energy-centric optimizations. The efficient operation of the final TLS4-3i and TLS2-3i designs is shown in Table 4. Specifically, on average 1.40 and 1.75 cores in TLS2-3i and $T L S 4-3 i$, respectively, are busy (Columns 2 and 3). Moreover, while busy, these cores execute instructions from squashed tasks for only $10.3 \%$ and $16.9 \%$ of the cycles, respectively (Columns 4 and 5). This is in contrast to NoOpt: on average, 2.00 cores are busy (Column 5 of Table 3 ), and $22.6 \%$ of the instructions executed belong to squashed tasks (Column 2 of Table 3).

\section{Related Work}

Past work on TLS CMP architectures has focused on performance rather than energy (e.g., $[9,10,13,21,22,25,26])$. There has been work on reducing the energy consumed in the pipeline due to instruction-level speculation following a branch prediction [2, 12]. However, the issues addressed are very different.

Concurrently to our work, Petric and Roth [16] developed an infrastructure for selecting pre-execution (prefetching) threads in an SMT processor. To select threads, they use models that minimize 


\begin{tabular}{||c||c|c|c|c||}
\hline \hline \multirow{2}{*}{ Apps } & \multicolumn{2}{|c|}{ Busy CPUs } & \multicolumn{2}{c||}{ Squashed Instr. (\%) } \\
\cline { 2 - 5 } & $T L S 2-3 i$ & $T L S 4-3 i$ & $T L S 2-3 i$ & $T L S 4-3 i$ \\
\hline \hline bzip2 & 1.17 & 1.36 & 4.6 & 7.5 \\
crafty & 1.46 & 1.68 & 17.0 & 18.2 \\
gap & 1.56 & 1.93 & 21.7 & 31.4 \\
gzip & 1.40 & 1.48 & 14.5 & 13.2 \\
mcf & 1.68 & 2.38 & 7.1 & 28.7 \\
parser & 1.10 & 1.25 & 6.4 & 13.9 \\
twolf & 1.29 & 1.62 & 1.7 & 4.4 \\
vortex & 1.31 & 1.49 & 6.5 & 7.8 \\
vpr & 1.58 & 2.58 & 12.9 & 27.2 \\
\hline Avg & 1.40 & 1.75 & 10.3 & 16.9 \\
\hline \hline
\end{tabular}

Table 4: Characterizing the optimized TLS4-3i and TLS2-3i chips. execution time, energy consumption, or $E \times D^{2}$. While their models are somewhat similar to those used in our TaskOpt optimization, the environments are very different. Our models are focused on trading off performance and energy in the event of a task squash. Such an event does not exist in their models. Unlike our tasks, their threads never get squashed, do not offload computation, are only spawned from the main thread, and are used in an SMT processor.

\section{Conclusions}

This paper refutes the claim that TLS consumes excessive energy and power. Its thesis is based on three contributions. The first one is identifying the main sources of energy consumption in TLS: task squashing, structures for data versioning and dependence checking, additional traffic due to these two effects, and additional instructions. The second contribution is proposing simple energy-saving optimizations to mitigate these sources. These optimizations cut the energy cost of TLS by over $60 \%$ on average, with minimal performance impact.

The third contribution is showing that the resulting TLS CMP offers a very desirable energy-performance trade-off, even for SPECint codes. A TLS CMP with four 3-issue cores delivers an average speedup of 1.27 over a 3-issue superscalar on full SPECint 2000 codes, while consuming only $25 \%$ more energy. Moreover, compared to a 6-issue superscalar with the same frequency, the TLS CMP is on average faster, while consuming only $85 \%$ of its total on-chip power, and yielding a $7.6 \%$ lower $E \times D^{2}$.

We hope that this work helps propel TLS into mainstream microprocessors. CMPs are attractive because they are more energyefficient, more scalable, and less complex than wide-issue superscalars. Moreover, they have an advantage for explicitly-parallel codes. In this paper, we showed that TLS CMPs can also speed up these most challenging SPECint codes, with lower power and energy consumption than wide superscalars. We expect better results for more parallel codes.

\section{REFERENCES}

[1] International Technology Roadmap for Semiconductors. Semiconductor Industry Association, 2002.

[2] J. L. Aragon, J. Gonzalez, and A. Gonzalez. Power-Aware Control Speculation Through Selective Throttling. In International Symposium on High-Performance Computer Architecture, pages 103-112, February 2003.

[3] D. Brooks, V. Tiwari, and M. Martonosi. Wattch: a Framework for Architectural-Level Power Analysis and Optimizations. In International Symposium on Computer Architecture, pages 83-94, June 2000.

[4] J. A. Butts and G. S. Sohi. A Static Power Model for Architects. In International Symposium on Microarchitecture, pages 191-201, December 2000 .

[5] M. Cintra, J. F. Martínez, and J. Torrellas. Architectural Support for Scalable Speculative Parallelization in Shared-Memory Multiprocessors. In International Symposium on Computer Architecture, pages 1324, June 2000.

[6] M. J. Garzarán, M. Prvulovic, J. M. Llabería, V. Viñals, L. Rauchwerger, and J. Torrellas. Tradeoffs in Buffering Memory State for Thread-Level Speculation in Multiprocessors. In International Symposium on HighPerformance Computer Architecture, pages 191-202, February 2003.
[7] SSA for Trees - GNU Project, May 2003. "http://www.gccsummit. org/2003/view_abstract.php?talk=2”.

[8] S. Gopal, T. Vijaykumar, J. Smith, and G. Sohi. Speculative Versioning Cache. In International Symposium on High-Performance Computer Architecture, pages 195-205, February 1998.

[9] L. Hammond, M. Willey, and K. Olukotun. Data Speculation Support for a Chip Multiprocessor. In International Conference on Architectural Support for Programming Languages and Operating Systems, pages 5869, October 1998.

[10] V. Krishnan and J. Torrellas. A Chip-Multiprocessor Architecture with Speculative Multithreading. IEEE Trans. on Computers, pages 866-880, September 1999.

[11] R. Kumar, K. Farkas, N. Jouppi, P. Ranganathan, and D. Tullsen. SingleISA Heterogeneous Multi-Core Architectures: The Potential for Processor Power Reduction. In International Symposium on Microarchitecture, December 2003.

[12] S. Manne, A. Klauser, and D. Grunwald. Pipeline Gating: Speculation Control for Energy Reduction. In International Symposium on Computer Architecture, pages 132-141, July 1998.

[13] P. Marcuello and A. Gonzalez. Clustered Speculative Multithreaded Processors. In International Conference on Supercomputing, pages 365372, June 1999.

[14] A. J. Martin, M. Nystroem, and P. Penzes. ET2: A Metric for Time and Energy Efficiency of Computation. Technical Report CSTR:2001.007, California Institute of Technology, December 2001.

[15] S. Palacharla, N. P. Jouppi, and J. E. Smith. Complexity-Effective Superscalar Processors. In International Symposium on Computer Architecture, June 1997.

[16] V. Petric and A. Roth. Energy-Effectiveness of Pre-Execution and Energy-Aware P-Thread Selection. Technical Report MS-CIS-03-34, University of Pennsylvania, November 2003.

[17] M. Prvulovic, M. J. Garzarán, L. Rauchwerger, and J. Torrellas. Removing Architectural Bottlenecks to the Scalability of Speculative Parallelization. In International Symposium on Computer Architecture, pages 204-215, June 2001.

[18] J. Renau. Chip Multiprocessors with Speculative Multithreading: Design for Performance and Energy Efficiency. $\mathrm{PhD}$ thesis, University of Illinois at Urbana-Champaign, 2004.

[19] J. Renau, J. Tuck, W. Liu, L. Ceze, K. Strauss, and J. Torrellas. Tasking with Out-of-Order Spawn in TLS Chip Multiprocessors: Microarchitecture and Compilation. In International Conference on Supercomputing, June 2005.

[20] P. Shivakumar and N. Jouppi. CACTI 3.0: An Integrated Cache Timing, Power and Area Model. Technical Report 2001/2, Compaq Computer Corporation, August 2001.

[21] G.S. Sohi, S.E. Breach, and T.N. Vijayakumar. Multiscalar Processors. In International Symposium on Computer Architecture, pages 414-425, June 1995.

[22] J. Steffan, C. Colohan, A. Zhai, and T. Mowry. A Scalable Approach to Thread-Level Speculation. In International Symposium on Computer Architecture, pages 1-12, June 2000.

[23] J. Steffan, C. Colohan, A. Zhai, and T. Mowry. Improving Value Communication for Thread-Level Speculation. In International Symposium on High-Performance Computer Architecture, February 2002.

[24] H. Su, F. Liu, A. Devgan, E. Acar, and S. Nassif. Full Chip Leakage Estimation Considering Power Supply and Temperature Variations. In International Symposium on Low Power Electronics and Design, August 2003.

[25] M. Tremblay. MAJC: Microprocessor Architecture for Java Computing. Hot Chips, August 1999.

[26] J. Tsai, J. Huang, C. Amlo, D. Lilja, and P. Yew. The Superthreaded Processor Architecture. IEEE Trans. on Computers, 48(9):881-902, September 1999.

[27] J. Tuck. A Novel Compiler Framework for a Chip-Multiprocessor Architecture with Thread-Level Speculation. Master's thesis, University of Illinois at Urbana-Champaign, 2004.

[28] H. S. Wang, X. P. Zhu, L. S. Peh, and S. Malik. Orion: A PowerPerformance Simulator for Interconnection Networks. In International Symposium on Microarchitecture, December 2002.

[29] Y. Zhang, D. Parikh, K. Sankaranarayanan, K. Skadron, and M. Stan. HotLeakage: A Temperature-Aware Model of Subthreshold and Gate Leakage for Architects. Technical Report CS-2003-05, University of Virginia, Department of Computer Science, March 2003. 